\title{
Programa computacional para aquisição de dados para avaliação de máquinas agrícolas ${ }^{1}$
}

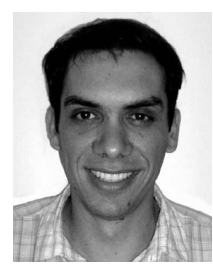

Ricardo F. Garcia ${ }^{2}$, Daniel M. de Queiroz ${ }^{3}$, Olímpio H. Miyagaki ${ }^{4} \&$ Francisco de A. de C. Pinto ${ }^{5}$

\author{
1 Parte da Tese de Doutorado do primeiro autor, apresentada no Programa de Pós-Graduação em Engenharia Agrícola, UFV \\ 2 DEA/UFV. CEP 36571-000, Viçosa, MG. Fone: (31) 3899-1877. E-mail: garcia@ufv.br (Foto) \\ ${ }^{3}$ DEA/UFV. Fone: (31) 3899-1882. E-mail: queiroz@ufv.br \\ ${ }^{4}$ UFV, Depto. de Matemática. Fone: (31) 3899-3011. E-mail: olimpio@ufv.br \\ ${ }^{5}$ DEA/UFV. Fone: (31) 3899-1881. E-mail: facpinto@ufv.br
}

Protocolo 172 - 3/12/2002 - Aprovado em 12/8/2003

\begin{abstract}
Resumo: A aquisição de dados é um dos principais problemas enfrentados por engenheiros e pesquisadores responsáveis pela realização de testes de máquinas agrícolas, devido à alta complexidade dessas máquinas. Para estabelecer os parâmetros relacionados ao seu desempenho, uma série de variáveis precisam ser monitoradas. Diante disso, o objetivo deste trabalho foi desenvolver um programa de computador para aquisição automática de dados a serem utilizados na avaliação de máquinas agrícolas. O sistema foi implementado utilizando-se o programa de computador LabVIEW versão 6i e equipamentos da "National Instruments". Desenvolveram-se Instrumentos Virtuais (VI) para os seguintes tipos de sensores: célula de carga, torquímetro, sensor de rotação tipo sensor indutivo e sensor de velocidade tipo radar. No trabalho realizado implementou-se um sistema de aquisição automática de dados e se desenvolveu o aplicativo AvaliaMA para avaliação de máquinas agrícolas, o qual foi desenvolvido buscando-se a coleta de dados de forma eficiente e se eliminando os erros tradicionais gerados pela coleta de dados convencional, como anotações incorretas em planilhas e perda de dados. Observou-se, durante os ensaios, facilidade de operação do sistema, permitindo rapidez na operação de aquisição de dados, e a capacidade de se combinar o sistema implementado para atender a demandas de diversas pesquisas, podendo ser utilizado em diferentes tipos de máquinas agrícolas.
\end{abstract}

Palavras-chave: automação, LabVIEW, mecanização, sensor

\section{Computer program for data acquisition to evaluate of agricultural machine}

\begin{abstract}
Data acquisition is one of the main problems that engineers and researchers face while testing agricultural machines, due to the high complexity of these machines. To analyze the performance of a machine, several variables need to be measured. The objective of this study was to develop an automatic data acquisition computer program for use in agricultural machine evaluation tests. The system was implemented using the LabVIEW software version 6i. Virtual instruments (VI) were developed for measuring data from the following sensors: load cell, torquimeter, rotational velocity meter and radar gun. The automatic data acquisition system developed showed, during the tests, capacity to present high precision results. It was found to be robust and versatile and has capacity to be combined in order to be used in different kinds of researches and conditions.
\end{abstract}

Key words: automation, LabVIEW, mechanization, sensor

\section{INTRODUÇÃO}

A aquisição de dados é uma atividade essencial em todo tipo de tecnologia e ciência. O objetivo de um sistema de aquisição de dados é apresentar, ao observador, os valores das variáveis, ou parâmetros, que estão sendo medidos. Geralmente, este valor numérico, ou medido, não é igual ao valor real da variável, ou seja, o sistema de aquisição tem como entrada o valor real da variável e sua saída é o valor medido.
Uma vez que a tomada de dados é uma das etapas mais tediosas de um ciclo de pesquisa, pode-se automatizar o sistema de aquisição sem comprometer a precisão, reduzindo os erros gerados pela transcrição de dados, eliminando as variações induzidas pelo operador nos processos de coleta de dados e aumentando a taxa de leitura de dados (Johnson, 1997).

Atualmente, a maioria de pesquisadores utiliza computadores pessoais (PC) com placas de expansão para aquisição de dados em pesquisas laboratoriais, controle industrial, e em 
testes e medições. Na agricultura, vários pesquisadores têm trabalhado em diferentes áreas, utilizando algum tipo de sistema de aquisição de dados com a finalidade de monitorar eventos em suas pesquisas. Al-Janobi (2000) desenvolveu um sistema de aquisição de dados baseado em transdutores de força e profundidade, para medir a força requerida e a profundidade de trabalho de implementos montados no sistema de três pontos do trator. Com o objetivo de operar automaticamente um pulverizador, Cho \& Lee (2000) desenvolveram um controlador "fuzzy", utilizando o receptor de um sistema de posicionamento global diferencial, para determinar a direção do percurso, e quatro sensores ultra-sônicos, para detectar os obstáculos durante a operação. Já Pedersen \& Pedersen (1995) utilizaram um sistema baseado em detectores infravermelhos passivos e interface de processamento de sinais analógicos para monitorar a atividade de animais domésticos, em campo. Também monitorando animais, Rometsch \& Becker (1993) empregaram um sistema de aquisição de dados portátil para armazenar, continuamente, os batimentos cardíacos de bois durante trabalho de tração.

A versatilidade de um sistema de aquisição também se torna importante, permitindo sua adaptação para diferentes pesquisas. Com o objetivo de medir a força de tração, a velocidade de deslocamento, a rotação e o torque da tomada de potência (TDP), a pressão e o fluxo do sistema hidráulico e a profundidade de trabalho, em tempo real, de implementos sob condições de campo, Arrivo \& Di Renzo (1998) desenvolveram um sistema de aquisição e um programa de processamento de dados, que se mostrou versátil e capaz de ser combinado para atender às demandas de pesquisas específicas.

A obtenção de resultados satisfatórios a partir de um sistema de aquisição baseado em um PC, depende do computador, de sensores, do condicionamento de sinais e dos equipamentos e programas de aquisição.

Nos equipamentos que se deseja monitorar, ou controlar, são instalados sensores que transformam um fenômeno físico em sinais elétricos. Os sinais elétricos produzidos variam de acordo com os parâmetros físicos que estão sendo monitorados, e devem ser condicionados para fornecer sinais apropriados à placa de aquisição de dados. Os acessórios de condicionamento de sinais amplificam, isolam, filtram e excitam sinais para que estes sejam apropriados às placas de aquisição. Uma vez condicionados e trabalhados na forma desejada, os sinais podem ser lidos em computadores, com placas específicas instaladas, e armazenados em diferentes formas, como arquivos de texto.

A configuração, o controle e o monitoramento da aquisição de dados, podem ser realizados com o auxílio de programas de computador específicos, como o LabVIEW (Laboratory Virtual Instrument Engineering Workbench) e equipamentos da "National Instruments", que trabalham dentro do conceito de instrumento virtual (VI). A instrumentação virtual tem como objetivo usar um computador comum para criar um aplicativo simulando instrumentos reais com controles e ambiente personalizados, mas com a versatilidade que acompanha o programa de aquisição (Johnson, 1997).

O LabVIEW é um ambiente de desenvolvimento de aplicativos, ou instrumentos virtuais, baseado em programação gráfica; utiliza terminologia, ícones e idéias familiares a técnicos, cientistas e engenheiros, e se baseia em símbolos gráficos ao invés de linguagem de texto para descrever ações de programação. Sua grande vantagem é que o usuário não necessita conhecer profundamente linguagens ou técnicas de programação para criar um instrumento virtual, ou aplicativo, fundamentado no conceito de fluxo de dados.

Utilizando o programa LabVIEW, Koontz (2001) desenvolveu um sistema para irrigação automatizada para ser utilizado por produtores tipicamente não técnicos, tornando-se uma eficiente ferramenta para monitorar e controlar o uso de água, reduzir custos e documentar a eficiência do uso da água no campo.

Thomopoulos (2001) utilizou o programa LabVIEW com a finalidade de desenvolver um sistema controlador automático para um dinamômetro, para controle e monitoramento de motores, capaz de fornecer operação manual e automática e monitorar, exibir e armazenar grande volume de dados, a taxas de leituras elevadas, referente ao desempenho do sistema.

Assim, o objetivo deste trabalho foi desenvolver um programa de computador para aquisição automática de dados a serem utilizados na avaliação de máquinas agrícolas, utilizando-se programas e equipamentos da "National Instruments", buscando-se a coleta de dados de forma eficiente e reduzindo os erros tradicionais gerados pela coleta de dados convencional.

\section{MATERIAL E MÉTODOS}

Este trabalho foi realizado no Laboratório de Mecanização Agrícola e no Laboratório de Projeto de Máquinas e Visão Artificial do Departamento de Engenharia Agrícola, na UFV. Esta etapa constou de instalação e configuração de sensores, de componentes e do programa LabVIEW versão $6 \mathrm{i}$, da "National Instruments". Foram utilizados os seguintes componentes:

-SCXI 1321 - bloco de terminais para conectar sinais provenientes dos sensores ao sistema de aquisição

-SCXI 1121 - módulo condicionador de sinais

-SCXI 1001 - chassi de suporte das placas do sistema de aquisição

-DAQCARD AI 16XE-50 - cartão tipo PCMCIA, inserido no computador portátil, para aquisição de dados multifuncional

-PSHR68-68M - cabo para conexão do cartão PCMCIA ao chassi

-SCXI 1349 - cabo SH6868 e adaptador de cabo

A operação de aquisição automática de dados envolveu os seguintes sensores: célula de carga modelo LCCA $10 \mathrm{~K}$ da Omega, com capacidade de $45 \mathrm{kN}$; torquímetro modelo TQ501$10 \mathrm{~K}$ da Omega, com capacidade de $1130 \mathrm{mN}$ e $6000 \mathrm{rpm}$; sensor tipo indutivo modelo PS5-18GI50-E da Sense; e sensor de velocidade modelo RVSII da Dickey-John. Foi utilizado, ainda, um computador portátil Compaq Presario para aquisição, processamento e armazenamento de dados.

O esquema de ligação dos componentes, sensores e computador portátil, é apresentado na Figura 1. 


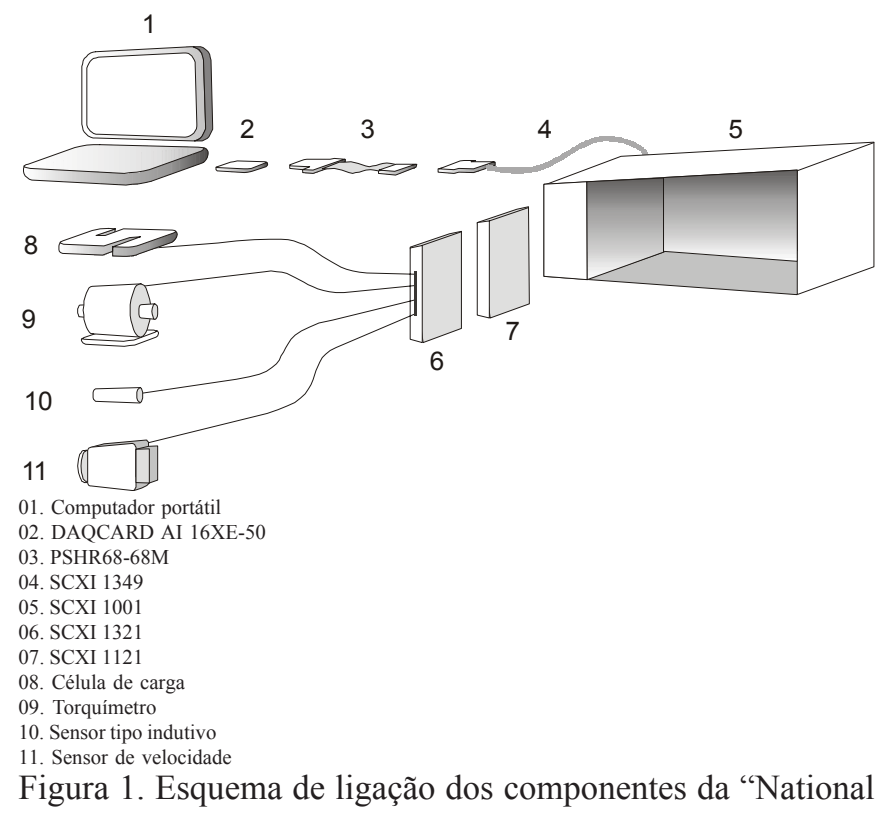

Instruments", sensores e computador portátil

A célula de carga converte uma força agindo sobre ela, em um sinal elétrico analógico através da deformação física de medidores de deformação, colados no bloco da célula de carga e ligados em forma de ponte de Wheatstone. Uma carga aplicada na célula, em compressão ou tensão, produz uma deflexão no bloco, que transmite deformação aos medidores. A deformação produz uma alteração na resistência elétrica proporcional à carga. Normalmente, uma voltagem de excitação é requerida na entrada e a saída pode ser lida em milivoltagem (Omega, 1992; Henry et al., 1991).

O torquímetro é constituído por um dispositivo de anel deslizante integral que é utilizado para transferir sinais elétricos da parte eletrônica rotativa para a parte eletrônica estacionária. $\mathrm{O}$ anel deslizante consiste de escovas de grafite que se encostam ao anel de rotação, fornecendo um percurso elétrico para a excitação de entrada e para a voltagem do sinal de saída de medidores de deformação ligados em forma de ponte de Wheatstone (Omega, 1992).

O sensor tipo indutivo é um equipamento eletrônico capaz de detectar a aproximação de peças metálicas, componentes, elementos de máquinas etc. A detecção ocorre sem que haja contato físico entre o sensor e o acionador, aumentando a vida útil do sensor, por não possuir peças móveis sujeitas a desgastes mecânicos. Tem como princípio de funcionamento a geração de um campo eletromagnético de alta freqüência, desenvolvido por uma bobina ressonante instalada na face sensora. A bobina faz parte de um circuito oscilador, gerando um sinal senoidal em condição normal e, quando esta é acionada com a proximidade de um metal, a energia do campo é absorvida pelo metal, diminuindo a amplitude do sinal gerado no oscilador. Esta variação é convertida em uma variação contínua que, comparada com um valor padrão, passa a atuar no estágio de saída (Sense, 2001; Bentley, 1995).

O radar é utilizado para medir a velocidade de deslocamento de um objeto, ou veículo, no qual foi instalado. Possui circuitos eletrônicos que emitem um sinal Doppler, em determinada freqüência, em direção ao solo. O sinal atinge um obstáculo e uma freqüência retorna a um circuito detector. Um microprocessador mede o período de cada ciclo detectado, processando-o e gerando uma freqüencia de saída correspondente a um número de pulsos por espaço percorrido (Dickey-John, 2001).

Utilizando-se o programa LabVIEW 6i, desenvolveu-se um VI, ou aplicativo, denominado AvaliaMA, para aquisição automática de dados com a finalidade de coletar, processar e armazenar dados provenientes dos sensores instalados numa máquina agrícola, especificamente uma colhedora de feijão.

O aplicativo AvaliaMA apresenta, aos usuários, os dados, enquanto lidos, em tela, na forma gráfica e numérica em tempo real; tem a função de criar e abrir um arquivo para armazenagem dos dados, adquirir dados provenientes de sensores a uma taxa e freqüência de leitura definida, realizar operações aritméticas com os valores lidos e armazenar os dados em arquivo de texto. $\mathrm{O}$ aplicativo desenvolvido inicia e continua a leitura, enquanto não ocorre erro interno do sistema de aquisição automática de dados, nem interrupção induzida pelo usuário.

O aplicativo AvaliaMA foi desenvolvido para ler os sinais dos sensores célula de carga e torquímetro, processando-os com a finalidade de obter a média em cada ciclo de leitura.

O aplicativo desenvolvido coleta os sinais do sensor de velocidade, numa taxa de aproximadamente 2900 amostras s $\mathrm{s}^{-1}$, tendo em cada ciclo 70 amostras lidas, e determina a freqüência do sinal numa função desenvolvida especificamente com esta finalidade. O aplicativo AvaliaMA coleta ainda os sinais do sensor indutivo, numa taxa de aproximadamente 90 amostras s ', tendo em cada ciclo 100 amostras lidas, e determina a freqüência do sinal numa função desenvolvida especificamente com este propósito.

O sistema de aquisição automática de dados, depois de implementado, foi testado preliminarmente com o objetivo de verificar a precisão dos dados provenientes dos sensores e calibrá-los. Para a calibração da célula de carga, foram utilizados pesos determinados em balança de precisão. Os pesos foram aplicados à célula de carga e os sinais gerados pelo sensor foram coletados e processados pelo sistema de aquisição automática de dados e comparados com os valores conhecidos aplicados, obtendo-se a equação de calibração do sensor.

$\mathrm{O}$ torquímetro foi montado sob uma base rígida, tendo um lado de seu eixo fixado e, no outro lado do eixo, foi montada uma porca travada com uma chaveta. Uma chave de boca, de comprimento determinado, foi acoplada à porca e pesos conhecidos foram aplicados na outra extremidade da chave. Os sinais gerados pelo sensor foram coletados e processados pelo sistema de aquisição automática de dados e comparados com os valores de torque calculados em função dos pesos aplicados e o comprimento do cabo da chave, obtendo-se a equação de calibração do sensor.

O sensor indutivo de proximidade foi montado próximo a uma peça metálica instalada no eixo de um motor elétrico. Variouse a rotação do motor e os sinais gerados pelo sensor foram lidos por um freqüencímetro, modelo Minipa APPA ET 2400, e também coletados e processados pelo sistema de aquisição automática de dados. Os dados do freqüencímetro e do sistema de aquisição de dados foram comparados, obtendo-se a equação de calibração do sensor. 
O sensor de velocidade foi montado no trator que teve sua velocidade teórica mensurada através do deslocamento em pista de teste, utilizando um cronômetro. Os sinais gerados pelo radar foram coletados e processados pelo sistema de aquisição automática de dados e comparados com os valores de velocidade teórica conhecidos, obtendo-se a equação de calibração do sensor.

As equações de calibração de cada sensor foram utilizadas na programação do aplicativo desenvolvido para efeito de correção dos dados obtidos pelo aplicativo; em seguida, o sistema de aquisição automática de dados foi testado em condições dinâmicas, comparando-se dados obtidos pelo aplicativo desenvolvido com dados lidos por outro sistema de leitura, conectado aos mesmos sensores. À célula de carga foram aplicadas diferentes forças de tração, usando-se dois tratores, sendo um para tracionar em pista de terra firme, e outro para ser rebocado com motor desligado em cinco diferentes marchas engatadas. Tomou-se a média de três repetições para cada marcha utilizada. Os dados obtidos pelo aplicativo AvaliaMA e os lidos por indicador digital, modelo Omega DP41-S, foram comparados.

$\mathrm{O}$ torquímetro foi instalado na árvore de acionamento de uma colhedora de feijão de arrasto, vazia, que foi acionada por um trator, utilizando-se cinco diferentes rotações da TDP, com três repetições. Os dados de torque obtidos pelo aplicativo e os lidos pelo indicador digital, foram comparados.

O sensor indutivo, instalado na TDP do trator, foi conectado ao sistema de aquisição automática de dados e ao freqüencímetro digital. Os dados obtidos pelo aplicativo e os lidos pelo freqüencímetro, foram comparados. Tomou-se a média de três repetições, utilizando-se cinco diferentes rotações.

$\mathrm{O}$ sensor de velocidade foi montado em trator que se deslocou em pista de terra firme, com $50 \mathrm{~m}$ de comprimento, utilizando-se cinco diferentes marchas, com três repetições. Os dados de velocidade de deslocamento obtidos pelo aplicativo e os determinados por medição direta, dividindo-se espaço por tempo, também foram comparados.

Após os ensaios preliminares, o sistema de aquisição automática de dados foi montado em uma máquina colhedora de feijão, tracionada por um trator, a fim de avaliar seu desempenho durante a operação de colheita de feijão. A operação de colheita foi realizada na Área Experimental da Epamig e no Laboratório de Automação Agropecuária da Embrapa Milho e Sorgo em Sete Lagoas, MG. O feijão, da variedade Pérola, foi utilizado nos ensaios de campo, sendo semeadas 12 a 13 sementes por metro linear, em espaçamento de $0,5 \mathrm{~m}$ entre linhas.

O trator utilizado no ensaio de campo foi o modelo MF 620 4 x 2, com tração dianteira auxiliar (TDA), da Massey Ferguson, enquanto a colhedora de feijão utilizada no mesmo ensaio foi a Double Master, produzida pelas Indústrias Reunidas Colombo Ltda. Esta colhedora é uma máquina do tipo arrasto, que trabalha acoplada à barra de tração do trator e possui um eixo com duas rodas de sustentação. A tomada de potência (TDP) do trator é utilizada para fornecer potência aos órgãos da máquina, como os sistemas de alimentação, trilha, separação, limpeza e transporte do produto ao tanque graneleiro.
A célula de carga foi montada na barra de tração do trator, num dispositivo construído de modo a eliminar forças laterais (Figura 2). Este dispositivo permite a leitura de forças apenas no sentido de tração e compressão. O torquímetro foi montado entre a árvore secundária de acionamento da colhedora e a árvore do cilindro trilhador da colhedora de feijão (Figura 3). O sensor indutivo foi montado na árvore de acionamento primária da colhedora de feijão (Figura 4). Nesta árvore, foi instalada uma peça metálica responsável por gerar os pulsos lidos pelo sensor. O sensor de velocidade foi montado na lateral do trator, na estrutura da escada do operador (Figura 5).

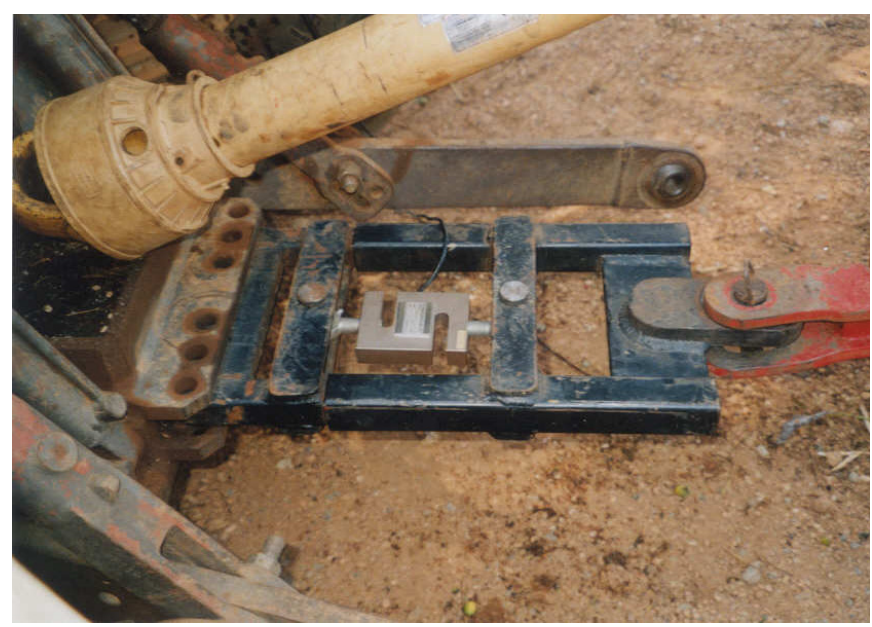

Figura 2. Detalhe de instalação da célula de carga montada na barra de tração do trator

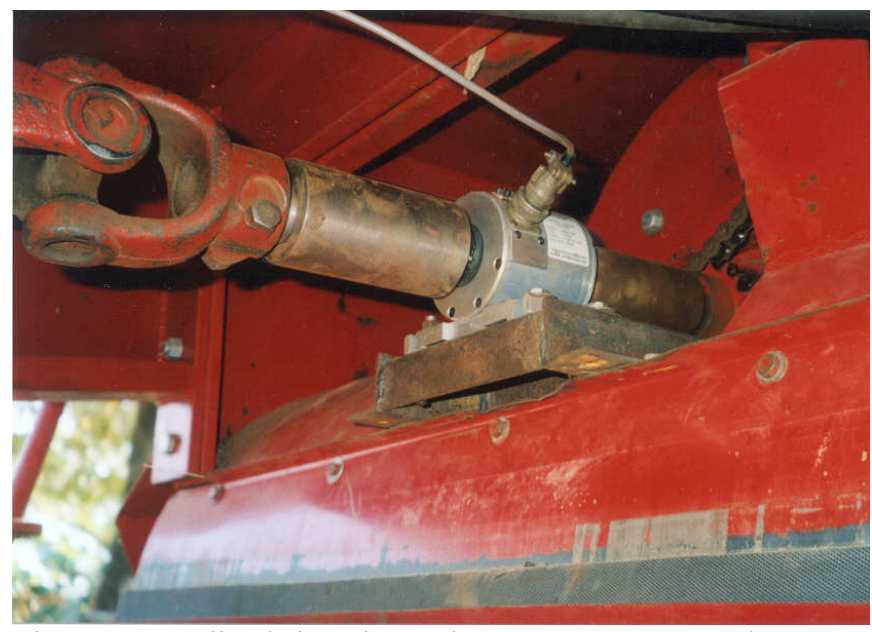

Figura 3. Detalhe de instalação do torquímetro montado entre a árvore secundária de acionamento da colhedora e a árvore do cilindro trilhador da colhedora de feijão

Os dados de força de tração requerida pela barra de tração, torque requerido e rotação da TDP, e velocidade de deslocamento da máquina colhedora de feijão, foram coletados, em tempo real, pelos sensores célula de carga, torquímetro, sensor indutivo e sensor de velocidade, respectivamente. $\mathrm{O}$ conjunto trator-colhedora de feijão foi ensaiado sob diferentes condições de operação, usando-se as velocidades de deslocamento de 4 , 7 e $10 \mathrm{~km} \mathrm{~h}^{-1}$, número de leiras de feijão colhido de 4,7 e 10 leiras, e rotação da TDP de 420 e 540 rpm. 


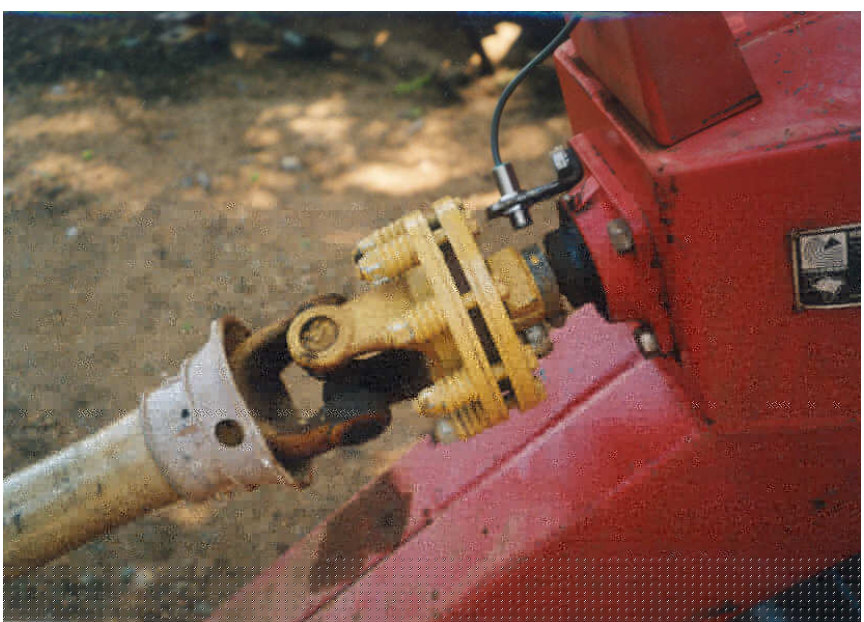

Figura 4. Detalhe de instalação do sensor indutivo montado na árvore de acionamento primária da colhedora de feijão

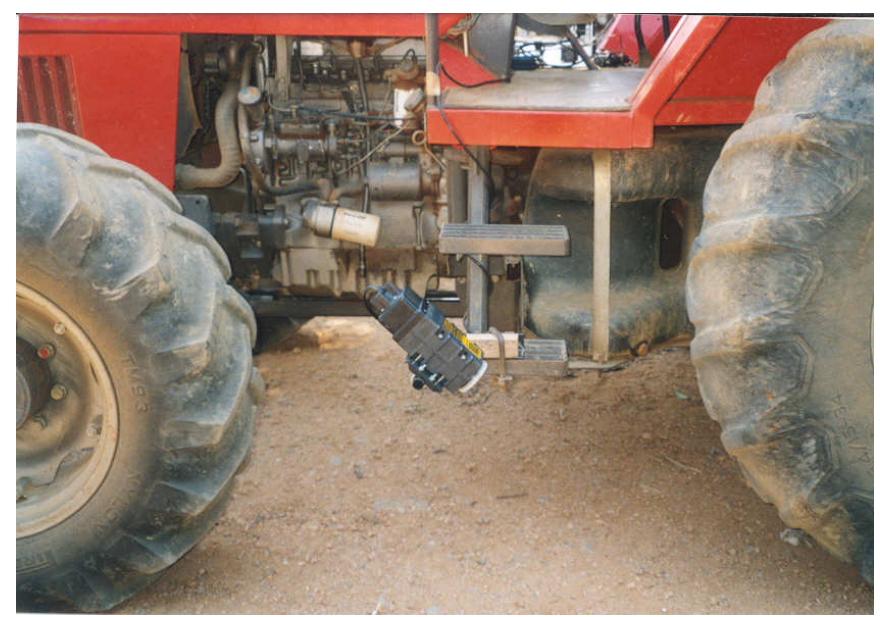

Figura 5. Detalhe de instalação do sensor de velocidade montado na lateral do trator

A potência requerida na barra de tração foi calculada a partir dos dados coletados de força de tração requerida na barra de tração e velocidade de deslocamento, conforme a Eq. 1.

$$
\text { ep } \mathrm{P}_{\mathrm{bt}}=\frac{\mathrm{Fv}}{3,6}
$$

em que:

$\mathrm{P}_{\mathrm{bt}}$ - potência requerida na barra de tração, $\mathrm{kW}$

$\mathrm{F}$ - força de tração requerida na barra de tração, $\mathrm{kN}$

$\mathrm{v} \quad$ - velocidade de deslocamento, $\mathrm{km} \mathrm{h}^{-1}$

A potência requerida na TDP foi calculada a partir dos dados coletados de torque requerido e a rotação da TDP, conforme a Eq. 2.

$$
\mathrm{P}_{\mathrm{tdp}}=\frac{\mathrm{Tn}}{9549,05}
$$

em que:

$\mathrm{P}_{\text {tdp }}$ - potência requerida na TDP, $\mathrm{kW}$

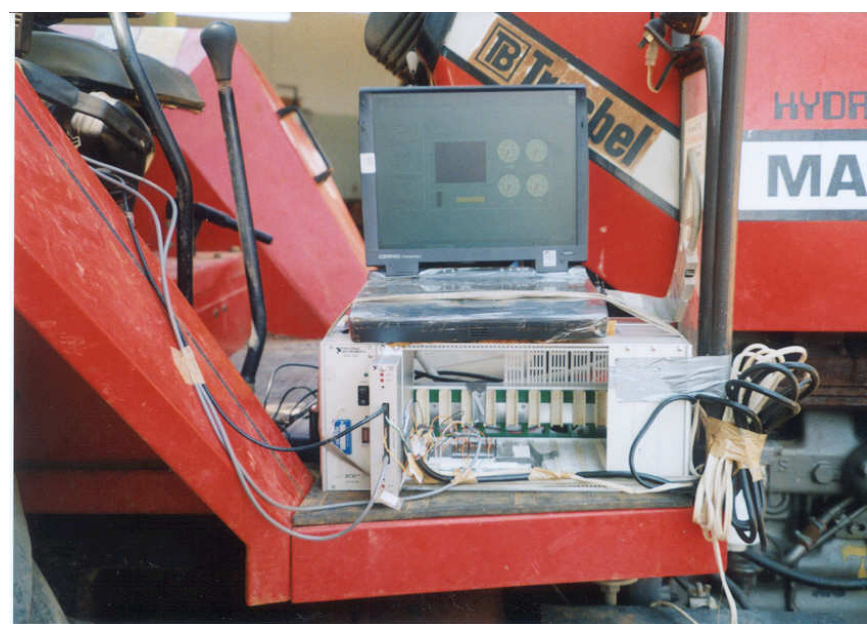

Figura 6. Vista geral do sistema de aquisição automática de dados

$$
\begin{array}{ll}
\mathrm{T} & \text { - torque requerido na TDP, } \mathrm{mN} \\
\mathrm{n} & \text { - rotação da TDP, rpm }
\end{array}
$$

\section{RESULTADOS E DISCUSSÃO}

Utilizando-se o programa LabVIEW 6i, desenvolveu-se um aplicativo denominado AvaliaMA, para aquisição automática de dados, com a função de apresentar os dados, enquanto lidos, em tela, na forma gráfica e numérica, em tempo real (Figura 7).

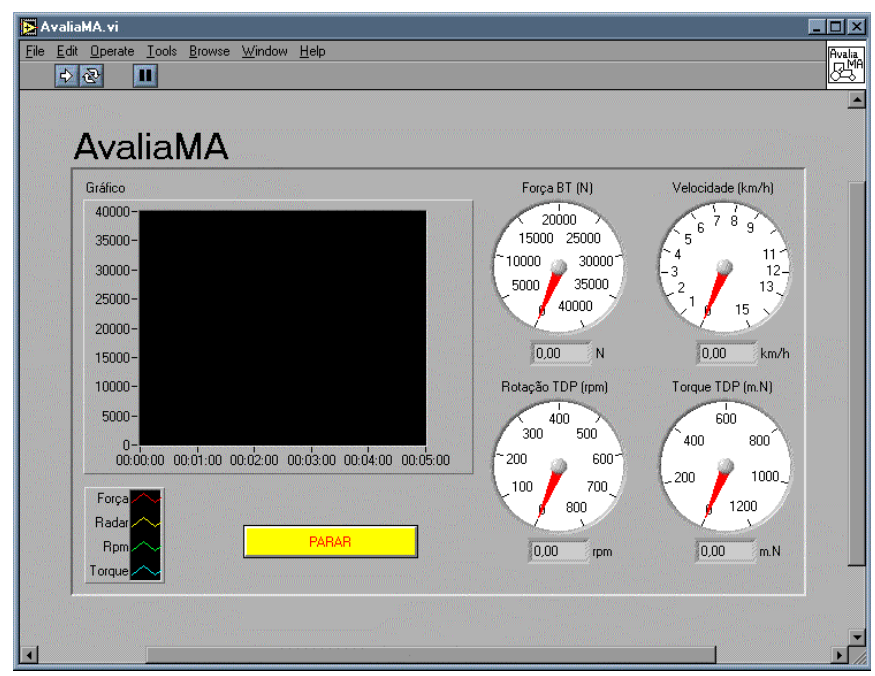

Figura 7. Tela de apresentação do aplicativo AvaliaMA

O aplicativo AvaliaMA foi programado para criar e abrir um arquivo para armazenagem dos dados, adquirir dados provenientes de sensores do tipo célula de carga, torquímetro, sensor indutivo e sensor de velocidade, a uma taxa e freqüência de leitura definida, realizar operações aritméticas com os valores lidos e armazenar os dados em arquivo de texto. $\mathrm{O}$ aplicativo desenvolvido inicia e continua a leitura, enquanto não ocorre erro interno do sistema de aquisição automática de dados nem na interrupção induzida pelo usuário (Figura 8).

O aplicativo AvaliaMA gera um arquivo de saída em formato doc em que os dados de saída são organizados em coluna (Tabela 1). 


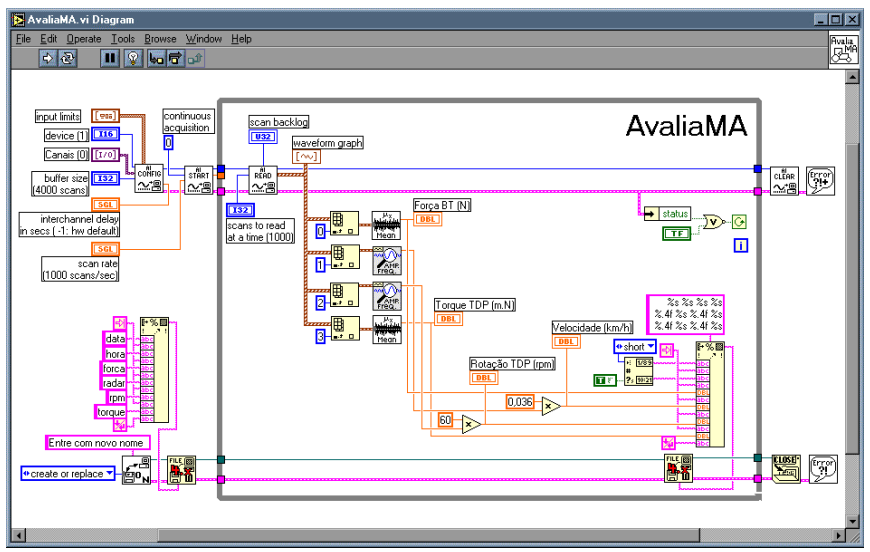

Figura 8. Tela de programação do aplicativo AvaliaMA

Tabela 1. Exemplo de arquivo de saída de dados do aplicativo AvaliaMA

\begin{tabular}{cccccc}
$\begin{array}{c}\text { Data } \\
(\mathrm{dd} / \mathrm{mm} / \text { aaaa })\end{array}$ & $\begin{array}{c}\text { Hora } \\
\text { (hh:mm:ss })\end{array}$ & $\begin{array}{c}\text { Força } \\
(\mathrm{kN})\end{array}$ & $\begin{array}{c}\text { Velocidade } \\
\left(\mathrm{km} \mathrm{h}^{-1}\right)\end{array}$ & $\begin{array}{c}\text { Rotação } \\
(\mathrm{rpm})\end{array}$ & $\begin{array}{c}\text { Torque } \\
(\mathrm{mN})\end{array}$ \\
\hline $06 / 09 / 2001$ & $11: 01: 11$ & 10,08 & 5,58 & 526,38 & 160,17 \\
$06 / 09 / 2001$ & $11: 01: 12$ & 10,07 & 5,67 & 520,07 & 160,77 \\
$06 / 09 / 2001$ & $11: 01: 13$ & 11,12 & 5,66 & 542,81 & 204,58 \\
$06 / 09 / 2001$ & $11: 01: 14$ & 11,41 & 5,77 & 534,74 & 216,36 \\
$06 / 09 / 2001$ & $11: 01: 15$ & 13,13 & 5,85 & 533,17 & 287,63 \\
$06 / 09 / 2001$ & $11: 01: 16$ & 16,11 & 5,66 & 538,83 & 411,08 \\
$06 / 99 / 2001$ & $11: 01: 17$ & 19,74 & 5,71 & 521,90 & 561,35 \\
$06 / 09 / 2001$ & $11: 01: 18$ & 20,35 & 5,75 & 525,37 & 585,69 \\
$06 / 09 / 2001$ & $11: 01: 19$ & 19,84 & 5,79 & 520,12 & 564,34 \\
$06 / 09 / 2001$ & $11: 01: 20$ & 18,89 & 5,67 & 526,35 & 525,08 \\
$06 / 09 / 2001$ & $11: 01: 21$ & 18,11 & 5,81 & 529,65 & 492,75 \\
$06 / 09 / 2001$ & $11: 01: 22$ & 17,25 & 5,72 & 538,71 & 457,22 \\
$06 / 09 / 2001$ & $11: 01: 23$ & 17,40 & 5,85 & 528,39 & 463,46 \\
$06 / 09 / 2001$ & $11: 01: 24$ & 19,08 & 5,84 & 524,05 & 533,06 \\
$06 / 09 / 2001$ & $11: 01: 25$ & 17,54 & 5,69 & 526,03 & 468,87 \\
$06 / 09 / 2001$ & $11: 01: 26$ & 14,90 & 5,45 & 528,80 & 359,92 \\
$06 / 99 / 2001$ & $11: 01: 27$ & 15,07 & 5,91 & 546,51 & 366,55 \\
$06 / 09 / 2001$ & $11: 01: 28$ & 15,42 & 5,77 & 531,06 & 381,54 \\
$06 / 09 / 2001$ & $11: 01: 29$ & 16,96 & 5,87 & 527,07 & 445,35 \\
$06 / 09 / 2001$ & $11: 01: 30$ & 16,47 & 5,66 & 530,41 & 424,22 \\
$06 / 09 / 2001$ & $11: 01: 31$ & 16,10 & 5,77 & 555,69 & 408,97 \\
$06 / 09 / 2001$ & $11: 01: 32$ & 14,56 & 5,96 & 525,34 & 345,86 \\
$06 / 09 / 2001$ & $11: 01: 33$ & 12,97 & 5,84 & 548,22 & 280,15 \\
$06 / 09 / 2001$ & $11: 01: 34$ & 12,68 & 5,72 & 525,39 & 268,11 \\
$06 / 09 / 2001$ & $11: 01: 35$ & 14,42 & 5,66 & 533,08 & 340,52 \\
$06 / 09 / 2001$ & $11: 01: 36$ & 14,71 & 5,78 & 526,45 & 352,21 \\
$06 / 99 / 2001$ & $11: 01: 37$ & 14,42 & 5,77 & 533,29 & 340,35 \\
$06 / 09 / 2001$ & $11: 01: 38$ & 14,73 & 5,99 & 540,23 & 352,71 \\
$06 / 09 / 2001$ & $11: 01: 39$ & 14,07 & 5,84 & 525,71 & 325,38 \\
\hline
\end{tabular}

Os sensores do tipo célula de carga, torquímetro, indutivo e de velocidade, foram conectados ao sistema de aquisição automática de dados implementados e calibrados em laboratório, cujos resultados são apresentados na Tabela 2.

O sistema de aquisição automática de dados foi testado em condições dinâmicas, comparando-se dados obtidos pelo aplicativo desenvolvido com dados lidos por outro sistema de leitura, conectado aos mesmos sensores. A comparação dos dados lidos pelo aplicativo AvaliaMA com os dados lidos por outro sistema de leitura, durante ensaios dinâmicos, é apresentada nas Figuras 9, 10, 11 e 12.

Analisando-se os gráficos de comparação dos ensaios dinâmicos dos sensores (Figuras 9, 10, 11 e 12) observa-se que os sinais obtidos pelo aplicativo, durante os ensaios, quando comparados com dados lidos por sistema de medição digital, apresentaram resposta linear.

Tabela 2. Equações de calibração dos sensores

\begin{tabular}{lcc} 
Sensor & \multicolumn{1}{c}{ Equação } & $\mathrm{R}^{2}$ \\
\hline Célula de carga & $\mathrm{y}=0,9906 \mathrm{x}-5,7084$ & 0,9999 \\
Torquímetro & $\mathrm{y}=0,9427 \mathrm{x}-0,1828$ & 0,9983 \\
Sensor indutivo & $\mathrm{y}=0,9978 \mathrm{x}+1,1668$ & 0,9999 \\
Sensor de velocidade & $\mathrm{y}=0,8217 \mathrm{x}+0,0094$ & 0,9992 \\
\hline
\end{tabular}

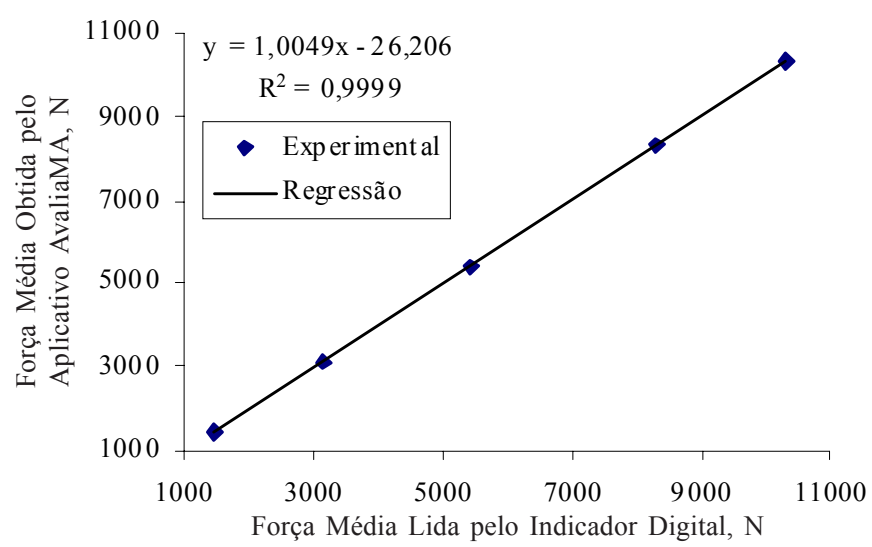

Figura 9. Comparação dos dados obtidos em ensaios dinâmicos da célula de carga

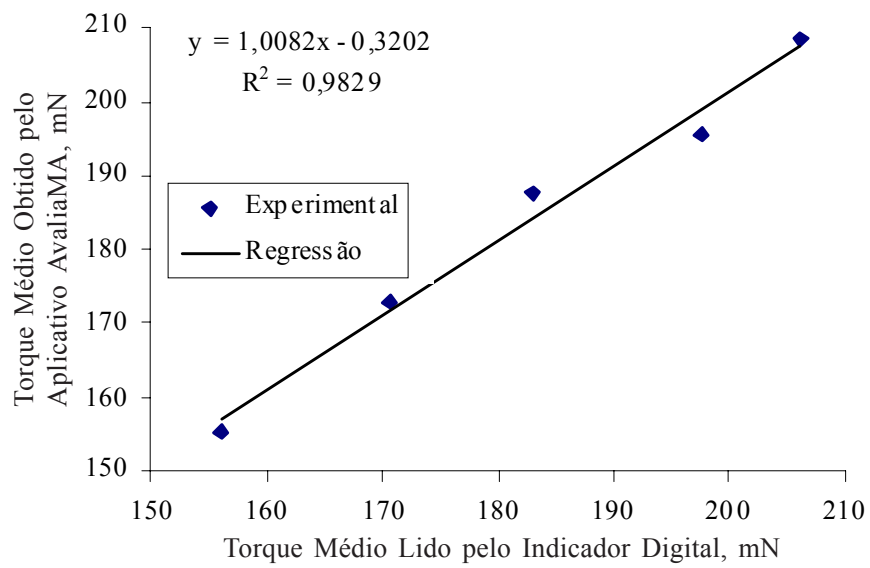

Figura 10. Comparação dos dados obtidos em ensaios dinâmicos do torquímetro

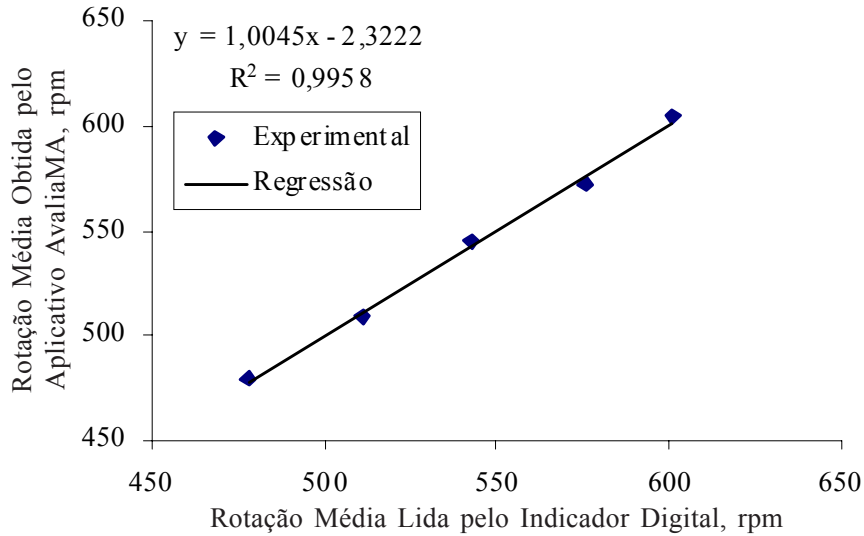

Figura 11. Comparação dos dados obtidos em ensaios dinâmicos do sensor indutivo 


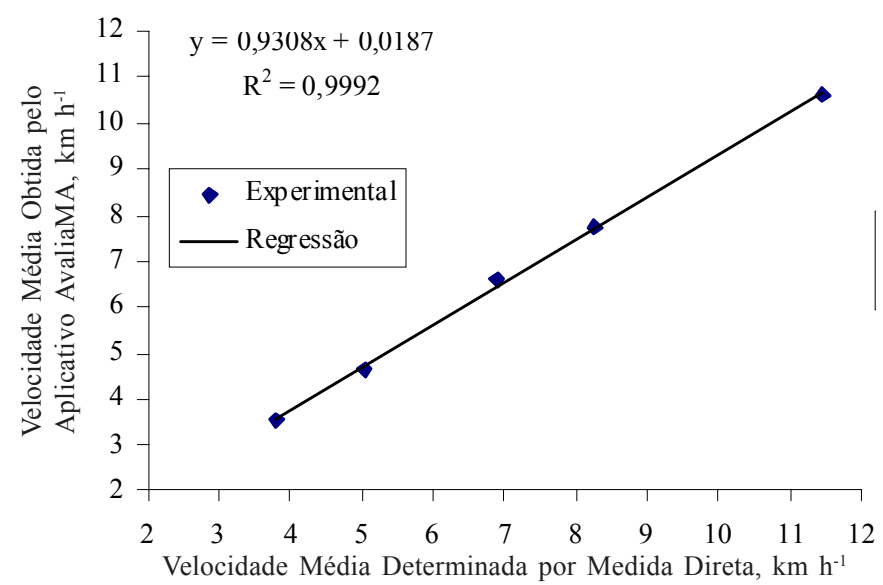

Figura 12. Comparação dos dados obtidos em ensaios dinâmicos do sensor de velocidade

\section{CONCLUSÕES}

1. No trabalho realizado implementou-se um sistema de aquisição automática de dados e se desenvolveu o aplicativo AvaliaMA, para avaliação de máquinas agrícolas.

2. O aplicativo AvaliaMA foi desenvolvido buscando-se a coleta de dados de forma eficiente e eliminando os erros tradicionais gerados pela coleta de dados convencional, como anotações incorretas em planilhas e perda de dados.

3. Comparando-se os resultados obtidos com o uso do AvaliaMA com as técnicas tradicionais, obteve-se um $\mathrm{R}^{2}$ superior a 0,98 , em todos os testes realizados, e se observou o maior erro relativo médio de $8,91 \%$, para a determinação de velocidade de deslocamento.

4. Observou-se, durante ensaios, facilidade de operação do sistema, permitindo rapidez na operação de aquisição de dados, e a capacidade de combinar o sistema implementado para atender a demandas de diversas pesquisas, podendo ser utilizado em diferentes tipos de máquinas agrícolas.

\section{AGRADECIMENTOS}

Ao Conselho Nacional de Desenvolvimento Científico e Tecnológico (CNPq), pela bolsa concedida. À Fundação de Amparo à Pesquisa do Estado de Minas Gerais (FAPEMIG) e ao Programa Redes Cooperativas de Pesquisa (RECOPE/FINEP), pelo auxílio à pesquisa e suporte financeiro. À EMBRAPA Milho e Sorgo e à EPAMIG de Sete Lagoas, pelo suporte na realização de testes experimentais. Às Indústrias Reunidas Colombo, pelo suporte financeiro.

\section{LITERATURA CITADA}

Al-Janobi, A. A data-acquisition system to monitor performance of fully mounted implements. Journal of Agricultural Engineering Research. London: Academic Press. v.75, n.2, p.167-175.2000.

Arrivo, A.; Di Renzo, G. C. Trailed unit for testing implements under field conditions. Journal of Agricultural Engineering Research. London: Academic Press. v.71, n.1, p.19-24. 1998.

Bentley, J.P. Principles of measurement systems. 3.ed. Singapore: Longman Singapore Publishers Ltd, 1995. 468p.

Cho, S. I.; Lee, J. H. Autonomous speedsprayer using differential global positioning system, genetic algorithm and fuzzy control. Journal of Agricultural Engineering Research. London: Academic Press. v.76, n.2, p.111-119, 2000.

Dickey-John. Radar II ground speed sensor. Disponível em: $<$ http://www.dickeyjohn.com/Ag_Products/Radar.htm>. Acesso em: 15 fevereiro 2001.

Henry, Z.A.; Zoerb, G.C.; Birth, G.S. Instrument and measurement for environmental sciences. 3.ed. St. Joseph, Michigan: ASAE, 1991.

Johnson, G.W. LabVIEW graphical programming: practical applications in instrumentation and control. New York: McGraw-Hill. 1997.665p.

Koontz, J.D. Using LabVIEW to automate commercial irrigation. National Instruments Corporation. Austin, Texas, 1995. Disponível em: $<$ http://digital.ni.com/csma.nsf/IntAllCSDocs/ 918665FFBA0EBFBF8625692D007898EB?OpenDocument\& node $=124200 \_$us $>$. Acesso em: 29 maio 2001.

Omega. The pressure strain and force handbook. Stamford: Ed. Omega Engineering, INC. v.28, 1992. 550p.

Pedersen, E.; Pedersen, C. B. Animal activity measured by infrared detectors. Journal of Agricultural Engineering Research. London: Academic Press. v.61, n.4, p.239-246. 1995.

Rometsch, M.; Becker, K. Determination of the reaction of heart rate of oxen to draught work with a portable data-acquisition system. Journal of Agricultural Engineering Research. London: Academic Press. v.54, n.1, p.29-36, 1993.

Sense. SENSE: sensores e instrumentos. Disponível em: $<$ http:/ /www.sense.com.br>. Acesso em: 15 fevereiro 2001.

Thomopoulos, P. Real-time dynamometer control with LabVIEW. National Instruments Corporation. Austin, Texas, 1996. Disponível em: $<$ http://digital.ni.com/csma.nsf/IntAllCSDocs/ 3D4448906DB5270E8625692D00789697?OpenDocument\& node $=124200 \_$US $>$. Acesso em: 29 maio 2001. 